\title{
STXM Study on Layered Nanomaterials: Graphene \& TMD
}

\author{
$\mathrm{J} \operatorname{Park}^{1 *}$ \\ 1. ETRI, Daejeon, Korea. \\ * Corresponding author: eureka99@etri.re.kr
}

Graphene exhibits unique mechanical, optical, and electronic properties, making it one of the most promising materials in prominent electronic devices [1]. To do so, it is essential to develop a method to synthesize large-scale high-quality graphene samples [2]. However, the most common growth technique, chemical vapor deposition (CVD), suffers from quality issues, which can arise from the hightemperature growth process as well as during the transfer process. In addition, chemical doping/cleaning process using plasma or wet chemistry further adds defects [3-4]. Therefore, in order to refine CVD graphene synthesis, transfer and post-treatment methods, it is important to effectively obtain information regarding defects and surface states during the process.

Scanning transmission $\mathrm{x}$-ray microscopy (STXM) is a promising experimental technique to study relevant physical and chemical information on graphene structure/defects by measuring the $\mathrm{C} 1 \mathrm{~s}$ nearedge $\mathrm{x}$-ray absorption fine structure (NEXAFS) which can give electronics/chemical states usually associated with different types of bonding and defects. In Fig. 1, we depict our preliminary data obtained for two different typical graphene samples (exfoliated a-few layer and CVD-grown/transferred monolayer graphene). The monolayer graphene was found to give enough signal-to-noise ratio for image and spectroscopic analysis. In the case of polymer-assisted transferred CVD graphene, we could observe some organic residues originating from the polymer coating and the non-flat non-uniform structural characteristics like wrinkle/folding which is not found in the exfoliated samples. The inherent chemical analysis based on the $\mathrm{x}$-ray spectro-microscopic method can identify the chemical species of the organic contaminants on the sample and the corresponding surface states of the surface-modified/functionalized sample which is very important for utilizing the graphene to various electronic, photonic and biooriented energy/sensing applications. By using these experimental fingerprints, we can reduce the damage and surface contamination during the post process. Therefore, our microscopic understanding will give a hint to prepare large-area graphene materials with controllable post-treatment for practical device applications.

\section{References:}

[1] KI Bolotin et al., Solid State Commun. 146 (2008), p. 351355.

[2] KS Kim et al., Nature 457 (2009), p. 706.

[3] L Zhao et al., Science 333 (2011), p. 999.

[4] B Guo et al., Nano Letters 10 (2010), p. 4975. 

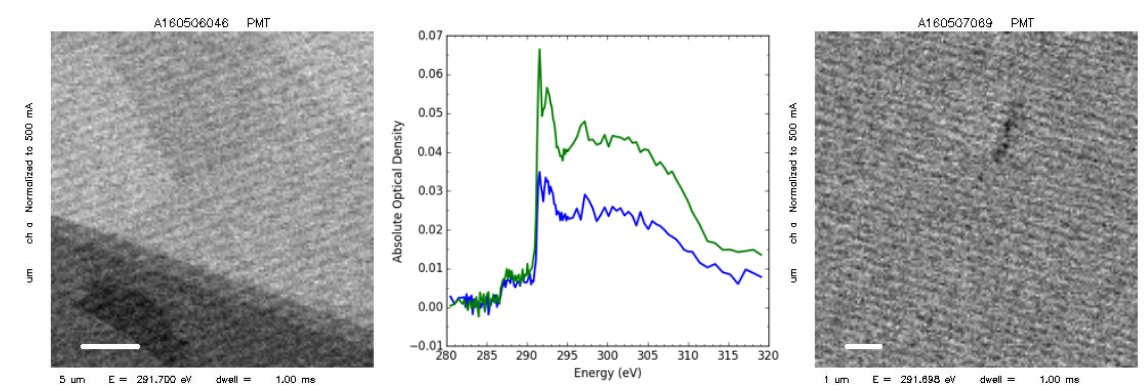

Figure 1. STXM data of exfoliated and CVD-grown monolayer graphene.
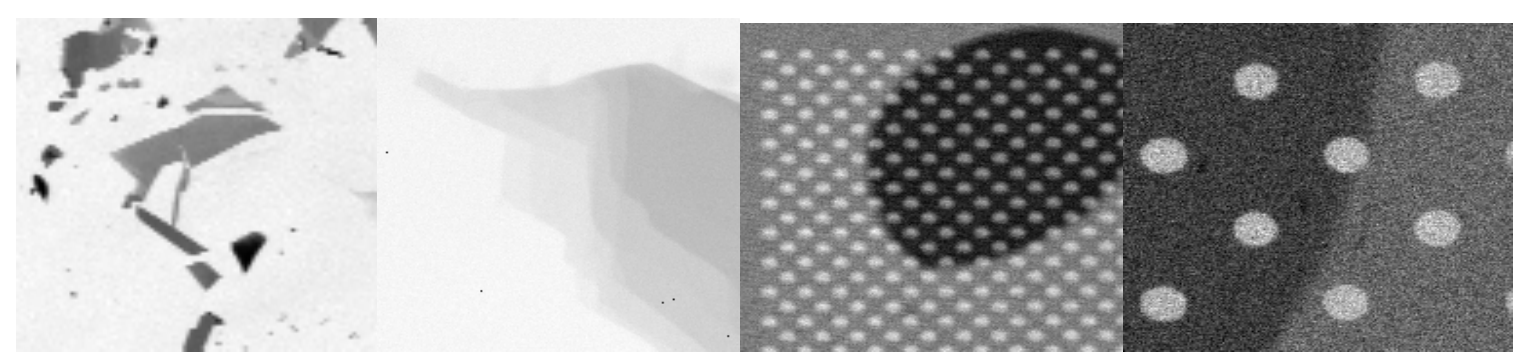

Figure 2. STXM data of exfoliated and MBE-grown TMD. 